\title{
Contribuições da avaliação neuropsicológica na qualificação dos sintomas do transtorno do espectro autista: Subsídios para o projeto terapêutico e educacional
}

\section{Contributions of neuropsychological assessment in the qualification of symptoms of autism spectrum disorder: Subsidies for the therapeutic and educational support}

DOI: $10.46919 / \operatorname{archv2n8-001}$

Recebimento dos originais: 01/11/2021

Aceitação para publicação: 31/12/2021

\section{Danillo Henrique de Jesus Reis}

Acadêmico em Psicologia - Universidade Federal de Goiás - UFG

Rua 235 - Setor Universitário - Goiânia - Goiás - CEP 74605-050

E-mail: danilloreis@live.com

\author{
Cláudia Santos Gonçalves Barreto Bezerra \\ Doutora em Ciências da Saúde - Universidade Federal de Goiás - UFG \\ Universidade Federal de Goiás - UFG \\ Rua Samambaia S/N - Chácaras Califórnia - Goiânia - GO - 74001-970 \\ E-mail: claudia_goncalves_barreto@ufg.br \\ Sandra de Fátima Barboza Ferreira \\ Doutora em Psicologia - PUC-GOIÁS \\ Universidade Federal de Goiás - UFG \\ Rua 235 - Setor Universitário - Goiânia - Goiás - CEP 74605-050 \\ E-mail: sandra_barboza@ufg.br
}

\begin{abstract}
RESUMO
O Transtorno do Espectro Autista - TEA caracteriza-se pelo comprometimento da comunicação social e presença de comportamentos repetitivos e estereotipados que conferem variados graus de prejuízo funcional. O objetivo desse trabalho é narrar o processo de avaliação neuropsicológica de uma criança com diagnóstico de TEA. Participou desse estudo um menino de seis anos, cursando o primeiro ano do ensino fundamental. Foram realizadas seis sessões de avaliação incluindo entrevistas com os pais, interlocução com a escola, observações do comportamento e administração de testes. Os resultados indicaram deficiência intelectual. A avaliação permitiu estabelecer o perfil cognitivo, bem como ampliar o conhecimento sobre interesses e pontos fortes no contato. Além da orientação para os pais indicaram-se condutas posteriores relacionadas ao apoio sistemático educacional, sobretudo no que diz respeito a adaptações nas atividades escolares, necessidade de pareamento concreto na apresentação destas e recursos lúdicos para alcançar minimamente o acesso à função simbólica.
\end{abstract}

Palavras-chave: atenção compartilhada, autismo, cognição, deficiência intelectual.

\begin{abstract}
The Autistic Spectrum Disorder - ASD stands out for the impairment of social communication and the presence of repetitive and stereotyped behavior that confer varying degrees of functional impairment. The aim of this work is to narrate the neuropsychological assessment process of a child diagnosed with ASD. A six-year-old boy attending the first year of elementary school participated in this study. Six sessions of evaluation were completed including interviews with parents, dialogue with the school, behavioral
\end{abstract}


observation, and test administration. The results indicated intellectual disability. The assessment made it possible to establish the cognitive profile, as well as to expand the knowledge about interests and strengths in interaction. In addition to orientations towards the parents, further conducts related to systematic educational support were indicated, especially regarding adaptations in school activities, the need for concrete pairing in the presentation of such activities and playful resources in order to minimally achieve access to the symbolic function.

Keywords: autism, cognition, intellectual disability, shared attention.

\section{INTRODUÇÃO}

O Transtorno do Espectro Autista - TEA é primariamente definido a partir do comprometimento da comunicação social e da presença de comportamentos repetitivos e estereotipados. Essa conceituação privilegia o caráter dimensional dos quadros clínicos em detrimento das tentativas de estabelecimento de fronteiras rígidas e claramente definidas entre os diagnósticos. (AMERICAN PSYCHOLOGICAL ASSOCIATION, 2014; BACKES, ZANON \& BOSA, 2017).

A adoção da nomenclatura espectro autista representa uma mudança paradigmática que privilegia a funcionalidade, uma vez que admite com maior clareza os diferentes níveis de comprometimento de um mesmo transtorno, possibilitando também a condução de uma atenção mais focalizada e efetiva. Esta conceituação permite que os diferentes grupos diagnosticados com o TEA possam ser definidos também segundo o grau de suporte que demandem (PAULA, CUNHA, SILVA E TEIXEIRA, 2017).

Embora muitos estudos busquem estabelecer uma forte base genética ao autismo, (ARBERAS e RUGGIERI, 2019), não existe um marcador biológico específico e o diagnóstico é estabelecido por critérios clínicos e com grande amparo dos manuais de classificação internacionais. Conforme os critérios apresentados pelo Manual diagnóstico e estatístico - DSM-5 (APA,2014), as primeiras manifestações sintomáticas devem aparecer antes dos 36 meses de idade. Porém é comum que o diagnóstico do TEA não se apresente com clareza até os 3 anos de idade, em função da sutileza dos comportamentos emitidos por indivíduos desta faixa etária (PAULA et al., 2017).

O TEA é hoje compreendido como um transtorno do neurodesenvolvimento que apresenta etiologias múltiplas e graus diversos de severidade e com ampla variação sintomatológica (PAULA ET AL., 2017; ROMEIRA; BOSA, 2021). O DSM-5 (APA, 2014) destaca que o TEA se caracteriza por prejuízos persistentes na comunicação e na interação social em múltiplos contextos, incluindo déficits na reciprocidade socioemocional, em comportamentos não verbais usados para interação e em habilidades para desenvolver, manter e compreender relacionamentos. Além dos déficits na comunicação social, o diagnóstico do transtorno do espectro autista requer a presença de padrões restritos e repetitivos de comportamento, interesses ou atividades, podendo ser mascarados por mecanismos compensatórios. Os 
critérios diagnósticos podem ser preenchidos com base em informações retrospectivas, embora a apresentação atual deva causar prejuízo significativo. (AMERICAN PSYCHIATRIC ASSOCIATION, 2014, p. 50-51).

O prejuízo na habilidade de Atenção Compartilhada (AC) é apontado pelas pesquisas recentes como o principal preditor do transtorno do espectro autista, se tornando, portanto, o foco mais importante para um diagnóstico diferencial (BOSA \& SALLES, 2018). O estudo experimental com crianças pré-escolares de Bosa (1998) demonstrou que, apesar de não se tratar de um comportamento completamente ausente nas crianças com autismo, a frequência da emissão de comportamentos de Atenção Compartilhada no grupo de referência foi substancialmente mais baixa do que no grupo controle. A variação se deu com base no contexto e no desenvolvimento global das crianças observadas.

De acordo com Bosa (2018), a atenção compartilhada (AC) é definida como a habilidade de coordenar a atenção entre dois parceiros sociais em relação a um terceiro, com vistas a compartilhar uma experiência. São comportamentos que apresentam propósito declarativo, pois envolvem vocalizações, gestos, contato ocular com a finalidade de "dividir" a experiência com as propriedades de objetos ou eventos ao redor (BOSA, 2002; BOSA, 2018). Por exemplo, a criança aponta para um objeto que está a certa distância e demonstra estar tentando mostrá-lo ao parceiro, alternando o olhar entre o objeto/evento e a face do parceiro. Frequentemente, esses gestos são também acompanhados por vocalizações e/ou expressões faciais. O desenvolvimento da $\mathrm{AC}$, imitação e brincadeira simbólica são fundamentais para o desenvolvimento global do indivíduo, pois se relacionam com a construção e aprimoramento da linguagem, do pensamento e da interação social.

O objetivo desse trabalho é narrar o processo de avaliação neuropsicológica de uma criança já diagnosticada pela equipe médica com TEA. Tendo em vista que crianças com esse diagnóstico raramente respondem a protocolos convencionais de avaliação, o que propor, alternativamente para viabilizar a avaliação? Buscou-se aporte teórico na Neuropsicologia (GLOZMAN, 2014; BOSA; TEIXEIRA, 2017; PONTRELLI MECA et al 2021).

\section{METODOLOGIA}

\subsection{CONTEXTO E DESCRIÇÃO DA DEMANDA}

A criança C. (6 anos) foi encaminhada à avalição neuropsicológica pelo serviço de Psicologia Escolar e pela Coordenação Pedagógica do colégio onde estuda, com vistas a mapear funções cognitivas que pudessem subsidiar o estabelecimento de condutas pedagógicas para maior adaptação e inclusão escolar. De acordo com informações da escola, ele apresenta dificuldades de permanecer em classe e acompanhar o ritmo da turma, demandando constante monitoramento. Ele demonstrou dificuldades significativas na comunicação e interação com os colegas e professores; ainda não conseguia desenvolver e manter relações apropriadas ao seu nível de 
desenvolvimento; padrões restritivos e repetitivos de comportamentos, interesses e atividades. Além disso o estudante apresentou comportamentos motores e vocais estereotipados e condutas sensoriais incomuns (preferência por materiais com cores fortes e contrastantes).

Em relação às habilidades escolares os professores observaram o interesse do aluno em folhear os livros literários e olhar as suas imagens, entretanto, não conseguia acompanhar a leitura do livro, mesmo de forma individualizada. Ia até a biblioteca de sala e pegava livros para tentar folheá-los e fitava as gravuras mais coloridas. Da mesma forma na biblioteca da escola. C. ainda não verbalizava nenhuma palavra, mas emitia sons demonstrando satisfação ou recusa. Na escrita começou a registrar pequenos rabiscos, mas faltava-lhe firmeza nas mãos.

Demonstrou interesse por músicas utilizadas em momentos de tarefas de sala aula e no laboratório de computação. Percebeu-se interesse especial pelos vídeos de músicas infantis ricos em cores contrastantes, provavelmente por enxergá-los melhor devido à baixa visão, segundo relato dos pais. Nas aulas de artes gostava de colar papéis coloridos e desenho livre. Nas aulas das outras disciplinas que requeriam mais atenção, precisava de maior incentivo para escrever, para colagem, ouvir histórias e assistir a vídeos curtos. Às vezes demonstrava impaciência, não tinha atenção suficiente para atividades na mesa da sala, precisava estar em movimento todo o tempo. Além disso, eventualmente apresentava comportamento inadequado de "morder os colegas" conduta que embora compreendida pelos colegas causava enorme desconforto na rotina escolar demandando suporte urgente e efetivo.

\subsection{PROCEDIMENTOS E INSTRUMENTOS}

Foram realizadas seis sessões com duração média de sessenta minutos e administradas as seguintes técnicas: Entrevista de Anamnese realizada com os pais; Registro anedótico (BENTZEN, 2012); Hora de Jogo Diagnóstica (EFRON, FAINBERG, KLEINER, SIGAL \& WOSCOBOINIK 2001; AFFONSO, 2012); Teste Não-Verbal de Inteligência - SON-R21/2 (TELLEGEN, LAROS, JESUS, KARINO, 2015)

Em função da impossibilidade de aplicação de uma bateria convencional, optou-se pela utilização de alguns procedimentos complementares: Avaliação Psicomotora (PICQ \& VAYER,1986); Sistema de Avaliação da Suspeita de Transtorno do Espectro Autista - PROTEA-R (BOSA \& SALLES 2018); Teste de triagem de Desenvolvimento - DENVER II (SABATÉS, LAMÔNICA, PERISSINOTO, BRÊTAS, SILVA, REZENDE, RESEGUE \& ISOTANI 2018). entrevista devolutiva para os pais e a escola. Projeto terapêutico/educacional. 


\section{RESULTADOS E DISCUSSÃO}

\subsection{CONDIÇÕES GERAIS DURANTE O PERÍODO AVALIATIVO}

De acordo com relatório médico o paciente tem diagnóstico de Transtorno do Espectro - TEA. Os pais ainda relatam que o Neuropediatra hipotetisa um quadro sindrômico, sendo o TEA parte das manifestações clínicas que ainda estão em investigação. O relatório Médico e prontuário referem Classificação Internacional de doenças - CID F84-9.

Durante todo o processo avaliativo o paciente esteve sob efeito de medicação anticonvulsivante: Valpakine. Em todas as sessões realizadas manteve um contato exploratório com o ambiente. Buscou contato com pessoas como meio para realizar objetivos. Foi capaz de interagir com os objetos que lhe são apresentados, contudo, aparenta ter maior interesse pelo aspecto sensorial dos mesmos do que por suas propriedades funcionais. Não demonstrou compreender instruções e apresentou com bastante frequência risos imotivados.

O paciente também demonstrou interesse por alguns objetos não convencionais do espaço físico da clínica: a mangueira de incêndio e as placas que indicam os extintores do corredor. Além disso, frequentemente explorou olfativamente objetos sintéticos e não o fazia como deveria se esperar com alimentos e outros produtos naturais. Apresentou alteração da acuidade visual (Estrabismo + hipermetropia). Embora haja prescrição médica de lentes corretivas, não faz uso frequente dos óculos e demonstrou desconforto tátil relacionado ao uso deles. Frequentemente apaga as luzes do ambiente e não parece fazê-lo como atitude meramente exploratória ou lúdica, demonstrando melhor conforto visual em ambiente com menos luminosidade.

\subsection{DADOS EXTRAIIDOS DA ANAMNESE}

Acompanhamento pré-natal a partir dos 3 meses de gestação; mãe relata uso de medicação para hipotireoidismo. Parto cesáreo, a termo. O paciente nasceu hipotônico e cianótico, mas não teve dificuldade de sucção; não necessitou de oxigenação suplementar; apresentou teste de Apgar 6/10. Recebeu alta hospitalar com 1 dia de vida. No período neonatal apareceram sintomas de refluxo. Apresentou atraso do desenvolvimento neuropsicomotor, com equilíbrio do pescoço aos oito meses, sentou-se com 12 meses e andou aos três anos e dois meses; nesta idade apresentou espasmos com EEG assintomático. A mãe ainda relatou uma internação por virose por volta de 1 ano e meio. C. ingressou na creche aos 3 anos; chegou a falar algumas palavras (pouco claras, mas que pareciam indicar referir-se à mãe e à solicitação de água) porém sua interação verbal regrediu. Em função dos atrasos apresentados, iniciou-se o processo de investigação neurológica. O controle dos esfíncteres ainda não foi estabelecido e a mãe relata uso diurno e noturno de fralda. Apresentou episódios de crises convulsivas com frequência irregular, controlados com uso de Valpakine iniciado aos 6 anos. Apresentou ranger de dentes ao dormir; auto e heteroagressividade; 
possui prejuízo significativo da visão. Há casos de hipermetropia acentuada na família e outros problemas de visão. Apresenta seletividade alimentar; alimenta-se exclusivamente de alimentos pastosos.

\subsection{DADOS EXTRAIIDOS DE EXAMES LABORATORIAIS E GENÉTICOS}

- Ressonância Magnética de Crânio aos 2 anos de idade - Pequena lesão focal ovalada sem realce pelo contraste na porção posterior do hemisfério cerebelar direito, inespecífica;

- Ressonância Magnética de Crânio aos três anos de idade - Pequena lesão focal córticosubcortical na porção posterior do hemisfério cerebelar direito, inespecífica, evidenciando aparecimento de pequenos focos de realce pelo contraste em relação ao exame de 17/06/2015. Este padrão evolutivo favorece a possibilidade de lesão primária mais remota;

- Diagnóstico Molecular da síndrome do X Frágil - Conclusão: “o resultado permite descartar a hipótese de sindrome do X-frágil;",

- Análise cromossômica por Microarray - Conclusão: “Am[hg19] 3p14.1(65,001,05965,529,996). Constituição cromossômica sexual XY. Foi identificada uma duplicação de aproximadamente $529 \mathrm{~kb}$ no braço curto do cromossomo 3, em 3p14.1. Nesta região está mapeado um gene OMIM (MAGI1 [602625]). Achado de significado incerto."

- $\quad$ Eletroencefalograma - EEG - Conclusão: EEG em sono evidenciando disfunção cortical paroxística potencialmente epileptogênica generalizada de predomínio focal temporal a direita, severa.

- $\quad$ Prontuário médico: conclusão: “Ao exame físico, apresenta estatura abaixo do percentil três, cabelos lisos, normoimplantados. Orelhas anteriorizadas, Estrabismo convergente, aparente hipotelorismo, FPs alongadas, cílios muito longos. Raiz nasal média, base média. Filtro nasolabial longo, lábio superior fino, comissuras orais desviadas para baixo. Queixo afilado. Pescoço sem alterações. Mãos e pés sem alterações dismórficas. Frouxidão ligamentar. Até o momento, não foi possível definir a etiologia das alterações apresentadas por C., que deve manter acompanhamento interdisciplinar. CID: F-84.9”.

- Relatório médico aos seis anos de idade: Atesta que o paciente "apresenta diagnóstico de autismo severo e epilepsia. Apresenta também atraso global do desenvolvimento, comprometimento cognitivo importante, não verbalização e hipotonia global. Apresenta grave prejuízo na interação social, relacionamento interpessoal e dificuldade de seguimento de instrução. Devido ao seu comprometimento cognitivo, não há previsão para vida independente."

\section{RESULTADOS QUANTITATIVOS E INTERPRETAÇÃO}

A tabela 1 apresenta os resultados obtidos pelo participante nos Subtestes do SON-R e os escores totais relativos aos escores de execução - EE e escores de raciocínio - ER, bem como o Quociente de 
inteligência - QI. Observa-se que os resultados se encontram substancialmente abaixo da média para o seu grupo etário identificando-se um atraso importante. Adicionalmente, como observação qualitativa pode-se destacar que não compreende a natureza das atividades, demonstra fraco engajamento em interações sociais com prejuízo importante da atenção compartilhada e não regula o comportamento a partir de instruções verbais. A figura 1 posiciona o participante em relação aos seus pares e indica a severidade do comprometimento cognitivo.

Tabela 1 - Teste não verbal de inteligência - SON - R 21/2 7

\begin{tabular}{|c|c|c|c|}
\hline Subteste & Escore & $\begin{array}{c}\text { Idade de } \\
\text { Referência }\end{array}$ & Escala \\
\hline Mosaicos & 0 & $<2,3$ & EE \\
\hline Categorias & 0 & $<2,3$ & ER \\
\hline Quebra-cabeças & 0 & $<2,3$ & EE \\
\hline Analogias & 0 & $<2,3$ & EE \\
\hline Situações & 0 & $<2,3$ & ER \\
\hline Escalas & Escore total & $\begin{array}{c}\text { Idade de } \\
\text { Referência }\end{array}$ & Percentil \\
\hline $\begin{array}{l}\text { Escalas de } \\
\text { Execução }\end{array}$ & 0 & $<2,0$ & $1 \%$ \\
\hline $\begin{array}{l}\text { Escala de } \\
\text { Raciocínio }\end{array}$ & 0 & $<2,0$ & $1 \%$ \\
\hline QI* & 0 & $<2,0$ & $1 \%$ \\
\hline
\end{tabular}

Figura 1- Posto percentil e Quocientes de inteligência nas escalas de raciocínio, execução e total. Fonte: SON-R
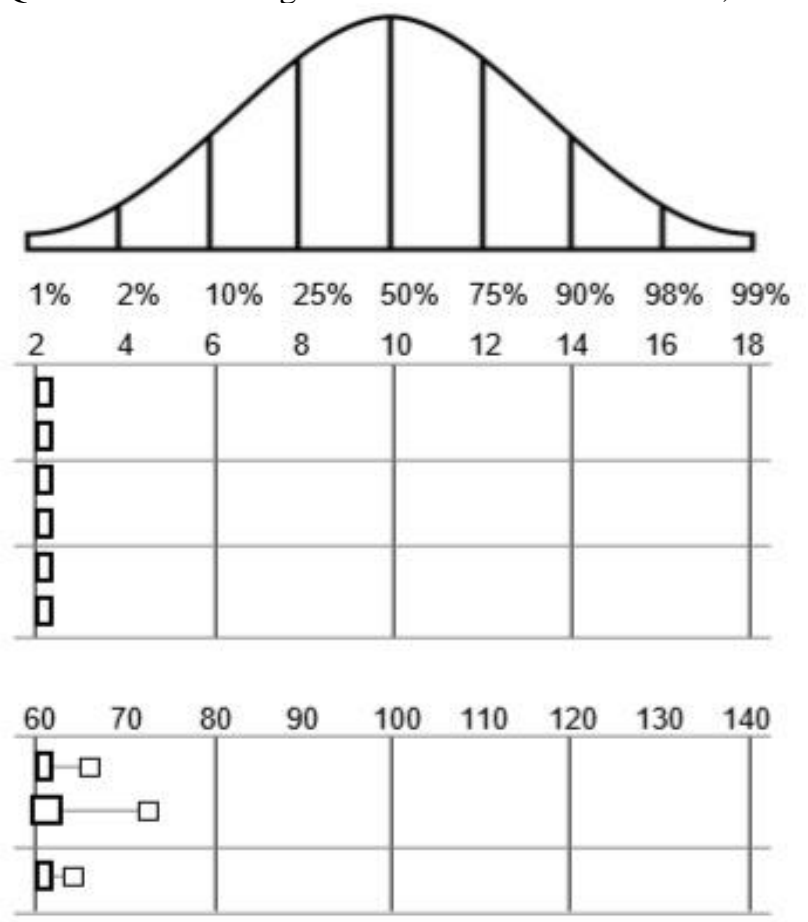
A figura 2 apresenta os resultados do participante no teste de triagem e desenvolvimento - DENVER II nos quatro domínios investigados relacionados aos aspectos sociais, motor fino e grosso e linguagem. Os resultados indicam atraso importante nos aspectos relacionados à linguagem e interação social que não atingem as condutas esperadas para o primeiro ano de vida e em relação a motricidade observa-se que o participante apresenta nível de realização em torno de 1 ano e sete meses. Além disso do ponto de vista observacional pode-se acrescentar que o desempenho é atípico, não cooperativo e exploratório.

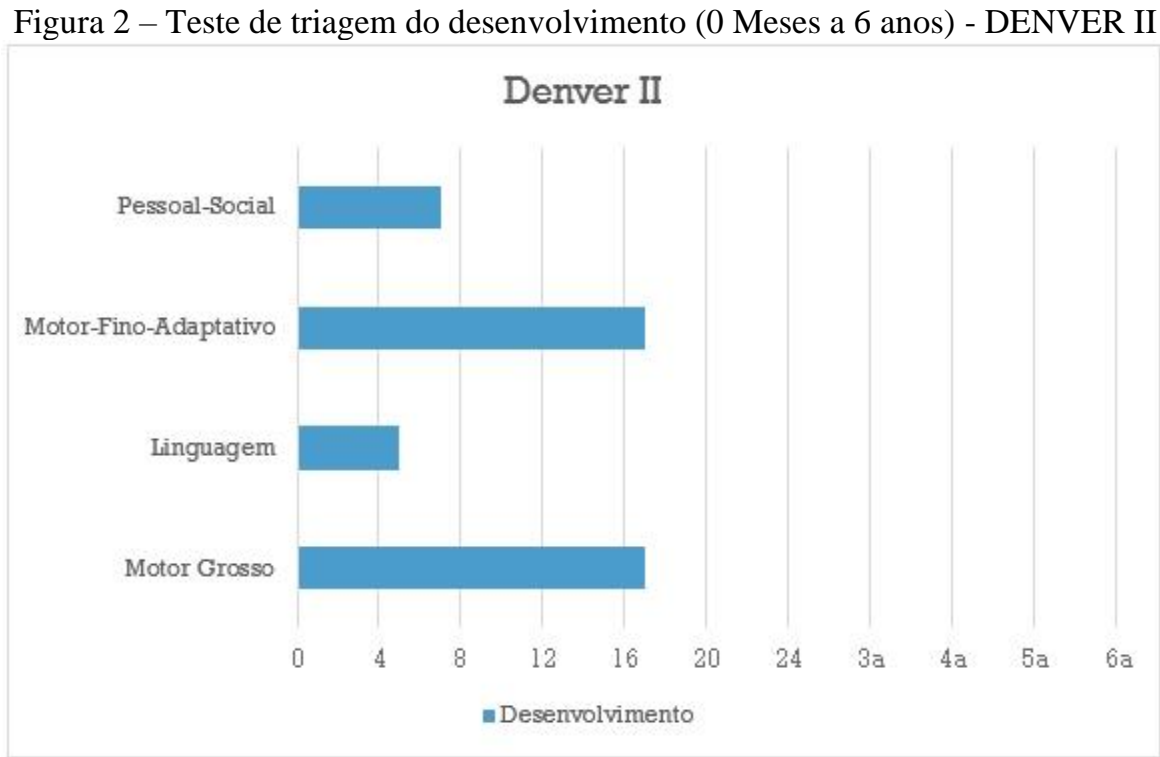

A figura 3 mostra os resultados apresentados na avaliação psicomotora nos seis domínios aferidos: coordenação dinâmica geral e dinâmica das mãos (ambidestro com discreta dominância direita), equilíbrio, controle do corpo, organização perceptiva e linguagem. Observa-se consistência com outros resultados aferidos na escala de desenvolvimento e teste de inteligência indicativos de atraso importante.

Figura 3 - Desempenho na avaliação psicomotora (Picq \& Vayer, 1988)

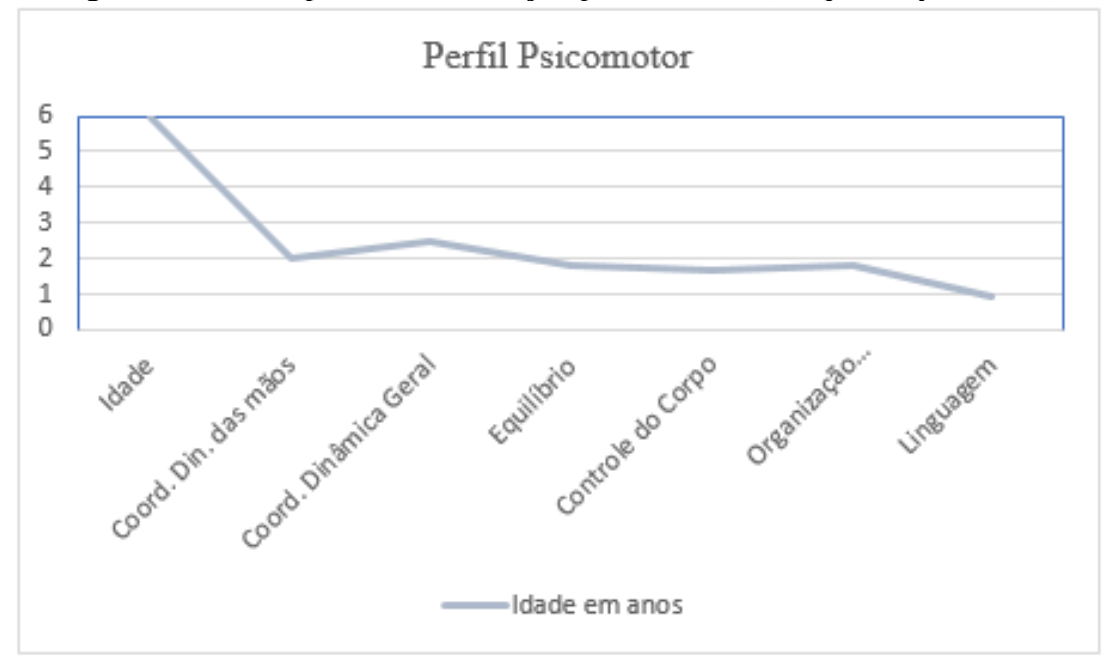


Qualitativamente ainda é possível destacar em relação a habilidades específicas atencionais e perceptivas que apresentou exacerbação da atenção difusa, tendo sua sustentação mais restrita a alguns objetos; demonstra intencionalidade ao direcionar o foco da atenção do adulto, mas não coordena com gestos, contato visual e expressões afetivas ou os gestos não são convencionais. Apresentou alteração da acuidade visual (Estrabismo + hipermetropia e hipotetisa-se fotossensibilidade). Reconhece objetos e parece sentir-se especialmente atraído pela cor vermelha. Gosta de música.

Apresentou habilidade mnemônica espacial (memória de deslocamento, recordando lugares anteriormente visitados) explorando lugares preferidos e implementando buscas a brinquedos, procurando por objetos em locais rotineiros; aparenta ser capaz de reconhecer pessoas com as quais mantém contato consistente (pais, irmão, monitores, terapeutas). Demonstra reconhecer personagens (Mickey, especialmente). É responsivo a músicas frequentemente tocadas.

Em relação á motricidade e praxias apresenta capacidade de preensão, habilidade de pinça, coordenação dos passos com intencionalidade. Apresenta dispraxia do vestir, ideatória, ideomotora e visoconstrutiva. Resolve problemas simples como abrir portas, apagar luzes, abrir caixas e retirar materiais. Reconhece contextos típicos para o comportamento de bater palma, reconhece situações de trabalho de mesa e compreende o uso típico de objetos de escrita, mas insistentemente tenta usar o adulto como um meio para realizar atividades.

A propósito das funções executivas apresenta déficits importantes de atenção compartilhada, inibição, planejamento, regulação e efetividade de ação. Por apresentar uma atenção difusa, precisa de mediação efetiva durante todas as atividades na escola.

\section{DISCUSSÃO}

Conforme espera-se em um quadro de TEA, o paciente apresentou déficits na reciprocidade socioemocional, compartilhamento reduzido de interesses, prejuízo de atenção compartilhada, comportamento simbólico comprometido e linguagem incipiente (eventualmente vocaliza) preenchendo critérios do DSM-V (APA, 2014, p.52) para TEA, gravidade nível 3 - "exigindo apoio muito substancial”.

O desempenho medido em testes padronizados bem como a observação da funcionalidade que aponta para prejuízos nas esferas da comunicação, interação social, auto-cuidado e habilidades acadêmicas ensejam a especificação do TEA “com comprometimento cognitivo” (APA, 2014, p.51). O acesso ao nível intelectual por meio do Teste Não verbal de inteligência (TELLEGEN, LAROS, JESUS, KARINO, 2015) no contexto do autismo tem sido recomendado (PONTRELLI MECA et al 2021). O atraso geral no desempenho intelectual $(\mathrm{QI}<47)$ merece ser qualificado para que possa subsidiar o projeto terapêutico. As dificuldades apresentadas indicaram principalmente a ausência de atenção compartilhada conforme destaca 
Bosa (2002) e Bosa e Fumagalli (2018). Mantém um contato exploratório com os materiais e não regula o comportamento a partir de instruções verbais.

Apresentou posicionamento heterogêneo em diferentes domínios do desenvolvimento aferidos por meio do Teste DENVER II (SABATÉS, LAMÔNICA, PERISSINOTO, BRÊTAS, SILVA, REZENDE, RESEGUE \& ISOTANI 2018); em relação à aquisição da linguagem e habilidades socioemocionais encontra-se posicionado em idade próxima aos seis meses de idade e no aspecto motor amplo e fino atinge idades em torno de um ano e seis meses, emitindo majoritariamente comportamentos relacionados ao período sensório-motor, nos quais a capacidade de acesso à função simbólica é incipiente. Quanto a isso algumas tarefas de pareamento concreto e nomeação foram sugeridas (BISTENE, 2019; BOSA; SALLES, 2018; ROGERS; DAWSON, 2010).

Em função da precária autonomia apresentada, sugeriu--se apoio substancial - supervisão ampla e permanente conforme preconiza o DSM-V (APA, 2014). Em relação à escolarização orientou-se considerar a possibilidade de frequentar múltiplas classes levando-se em consideração as tarefas que se encontram na zona de desenvolvimento proximal (Este conceito deriva da teoria Vygotskyana (VIGOTSKI, 1998; ALVAREZ; DEL RIO, 1996) e se define pela distância existente entre o nível de desenvolvimento real e o nível de desenvolvimento potencial do sujeito mantendo-o sempre que possível em contato com seus pares.

Conforme preconizam as diretrizes de atenção ao TEA (BRASÍLIA, 2014) indicou-se ainda Atendimento Educacional Especializado; Continuidade da Fonoterapia; Psicoterapia especializada: ABA, HANEM, FLOORTIME, SON-RISE. TEACH; Terapias de apoio: Musicoterapia, Psicoterapia, Terapia ocupacional; Adaptações curriculares no nível da metodologia e dos objetivos (CAPELLINI, 2018). Orientações à família são altamente necessárias e desejáveis e compõem o conjunto de medidas de suporte ao tratamento (PAULA ET AL, 2017; SOUZA; PINHEIRO; MACHADO 2021).

Destaca-se que a avaliação neuropsicológica cumpre uma função diagnóstica no sentido de qualificar os sintomas (LURIA, 1991), estabelecer o perfil neuropsicológico (CARVALHO; FERREIRA; SCHWARTZMAN; SEABRA, 2021) a fim de subsidiar o projeto terapêutico/habilitação (GLOZMAN, 2014).

\section{CONSIDERAÇÕES FINAIS}

A avaliação permitiu estabelecer o perfil cognitivo, concluindo tratar-se de um quadro combinado com deficiência intelectual severa e acentuados prejuízos de funcionalidade adaptativa no que se refere à interação social e comunicação, autocuidado e desempenho acadêmico, identificando as dificuldades de atenção compartilhada, características do TEA.

A avaliação também indicou condutas posteriores relacionadas à necessidade de terapias especializadas já fortemente consolidadas na literatura e encaminhamentos de apoio sistemático 
educacional, sobretudo no que diz respeito à organização e apresentação de conteúdos didáticos mediados por pareamento concreto e recursos lúdicos para alcançar minimamente o acesso à função simbólica.

Foram sugeridos os seguintes encaminhamentos: retorno ao oftalmologista para investigação da fotossensibilidade e aprofundamento da investigação genética. Idealmente a realização periódica da avaliação neuropsicológica pode monitorar o efeito e efetividade das estimulações clínicas e educacionais bem como regular e reorientar o projeto terapêutico. 


\section{REFERÊNCIAS}

AFFONSO, R. M. L. (Org.). Ludodiagnóstico: investigação clínica através do brinquedo. São Paulo: Artmed, 2012.

AMERICAN PSYCHIATRIC ASSOCIATION. Manual Diagnóstico e Estatístico de Transtornos Mentais - DSM 5. Porto Alegre: Artes médicas, 2015.

ALVAREZ, A; DEL RIO. Pablo. Educação e Desenvolvimento: A teoria de Vygotsky e a Zona do Desenvolvimento Próximo. In: COLL, C.; PALÁCIOS, J.; MARCHESI. Desenvolvimento Psicológico e Educação. Psicologia da Educação. Vol.2. Porto alegre: ARTMED, 1996.

ARBERAS, Claudia; RUGGIERI, Víctor. Autismo: Aspectos genéticos y biológicos. Medicina (B. Aires), Ciudad Autónoma de Buenos Aires, v. 79, n. 1, supl. 1, p. 16-21, abr. 2019. Disponible en $<$ http://www.scielo.org.ar/scielo.php?script=sci_arttext\&pid=S0025-

$76802019000200005 \& \operatorname{lng}=e s \& n r m=i s o>$. Acesso em $21 \mathrm{dez} 2019$.

BACKES, Bárbara; ZANON, Regina Basso; BOSA, Cleonice Alves. Características Sintomatológicas de Crianças com Autismo e Regressão da Linguagem Oral. Psic.: Teor. e Pesq., Brasília, v. 3333-3343, 2017. Available from <http://www.scielo.br/scielo.php?script=sci_arttext\&pid=S010237722017000100403\&lng=en\&nrm=iso>. Acesso em 21 Feb. 2020. Epub June 22, 2017. https://doi.org/10.1590/0102.3772e3343.

BENTZEN, Warren. Guia para observação e registro do comportamento infantil. São Paulo: Cengage learning, 2012.

BISTENE, Marta. Kit Autismo: Habilidades cognitivas, 2019.

BOSA, Cleonice Alves. Affect, communication, and self-stimulation in children with and without autism: a systematic observation study of joint attention and behaviours. Londres: Universidade de Londres, 1998.

BOSA, Cleonice Alves. Atenção compartilhada e identificação precoce do autismo Psicologia: Reflexão e Crítica, 2002.

BOSA, Cleonice Alves; BACKES, Barbara, ROMEIRO, Gabriela e ZANON, Regian Basso. Avaliação sociocomunicativa nos casos de suspeita de autismo: diretrizes para a hora lúdica diagnóstica. In: BOSA, Cleonice Alves; TEIXEIRA, Maria Cristina trigueiro veloz. (Orgs). Autismo: Avaliação psicológica e neuropsicológica. São Paulo: hogrefe. 2017.

BOSA, Cleonice Alves; SALLES, Jerusa Fumagalli. PROTEA-R Sistema de Avaliação de Suspeita de Transtorno do Espectro Autista. São Paulo: Vetor Editora, 2018.

BRASÍLIA. Diretrizes de Atenção à Reabilitação da Pessoa com Transtornos do Espectro do Autismo (TEA). Brasília, 2014.

CARVALHO, A. C. R..; FERREIRA, S. de F. B.; SCHWARTZMAN, J. S..; SEABRA, A. G. . Perfil neuropsicológico de um adolescente com síndrome de potocki-lupski (duplicação 17p11. 2 p11. 2): estudo de caso: Neuropsychological profile in an adolescent with potocki-lupski syndrome: case study. Archives 
of Health, [S. l.], v. 2, n. 5, p. 1465-1476, 2021. DOI: 10.46919/archv2n5-008. Disponível em: https://latinamericanpublicacoes.com.br/ojs/index.php/ah/article/view/724. Acesso em: 11 nov. 2021.

CAPELLINI, Vera Lúcia Messias Fialho. Adaptações curriculares na inclusão escolar. Curitiba: Appris, 2018.

MECCA, Tatiana Pontrelli e DIAS, Natália Martins. Cognição social no Transtorno do espectro autista. In: BOSA, Cleonice Alves; TEIXEIRA, Maria Cristina trigueiro veloz. (Orgs). Autismo: Avaliação psicológica e neuropsicológica. São Paulo: hogrefe. 2017.

DIAS, S. Asperger e sua síndrome em 1944 e na atualidade. São Paulo: Revista Latinoamericana de Psicopatologia Fundamental, 2015.

EFRON, A. M.; FAINBERG, E. KLEINER, Y.; SIGAL, A. M.; WOSCOBOINIK, P. A hora de jogo diagnóstica. In: OCAMPO, M. L. S; ARZENO, G.; PICCOLO, E. G. (Orgs.). O processo psicodiagnóstico e as técnicas projetivas. São Paulo: Martins Fontes, 1981, p. 167-202.

GLOZMAN, Janna. A prática neuropsicológica fundamentada em Luria e Vygotsky: avaliação, habilitação e reabilitação na infância. Tradução: Carla Anauate. São Paulo: Memnon, 2014.

FERNANDES, F.D.M. A questão da linguagem em autismo infantil: uma revisão crítica da literatura. Rev. Neuropsiq da Inf. Adolesc. 1994;2(3):5-10.

GADALLA KK, BAILEY ME, COBB SR. MeCP2 and Rett Syndrome: revesibility and potential avenues for therapy. Biochem J. 2011; 439(1):1-14. 22.

LAROS, J. A., TELLEGEN, P. J., JESUS, G. R., \& KARINO, C. A. (2015). SON-R 2V2-7[aJ. Manual Teste não-verbal de inteligência. São Paulo, SP: Hogrefe.

LURIA, Alelsandr Romanovich. Fundamentos de Neuropsicologia. São Paulo: livros Técnicos e Científicos: 1991.

MERCADANTE, Marcos T; VAN DER GAAG, Rutger J; SCHWARTZMAN, Jose S. Transtornos invasivos do desenvolvimento não-autísticos: síndrome de Rett, transtorno desintegrativo da infância e transtornos invasivos do desenvolvimento sem outra especificação. Rev. Bras. Psiquiatr., São Paulo, v. 28, supl. 1, p. s12- s20, May 2006.

MIILHER, Liliane Perroud; FERNANDES, Fernanda Dreux Miranda. Habilidades pragmáticas, vocabulares e gramaticais em crianças com transtornos do espectro autístico. Pró-Fono R. Atual. Cient., Barueri, v. 21, n. 4, p. 309-314, Dec. 2009. Available from.

PAULA, C.S., CUNHA, G.R., SILVA, L.C., \& TEIXEIRA, M. Conceituação do Transtorno do Espectro Austista: definição e epidemiologia. Hogrefe, São Paulo: Autismo: avaliação psicológica e neuropsicológica, 2017.

PONTRELLI MECCA, T.; MANUELLY FEITOSA DE LIMA, R.; ARIE LAROS, J.; COUTINHO DE MACEDO, E.; LOWENTHAL, R. Transtorno do Espectro Autista: Avaliação de habilidades cognitivas utilizando o teste não-verbal SON-R 6-40. Psicologia: Teoria e Pesquisa, [S. l.], v. 36, 2020. DOI: $10.1590 / 0102.3772 \mathrm{e} 3624$. 
PICQ, L. e VAYER, P. Educação Psicomotora e Retardo Mental. Porto Alegre: Artes Médicas, 1986.

ROGERS, Sally; DAWSON, Geraldine. Intervenção Precoce em crianças com Autismo. Liboa: LIDEL (2014).

ROMEIRA, Gabriela Moreira; SCHREINER, Letícia Backes; BOSA, Cleonice Alves. Avaliação Psicológica de Crianças com Suspeita de TEA: Perfil Interativo dos Avaliadores. Aval. psicol., Campinas, v. 20, n. 1, p. 43-51, mar. $2021 . \quad$ Disponível em <http://pepsic.bvsalud.org/scielo.php?script=sci_arttext\&pid=S167704712021000100006\&lng=pt\&nrm=iso>. acessos em jul. 2021. http://dx.doi.org/10.15689/ap.2021.2001.19578.05.

SABATÉS, A.L; LAMÔNICA, D.A.C.; PERISSINOTO, J.; BRÊTAS, J.S.; SILVA, M.G.B.; REZENDE, M.A.; RESEGUE, R.F.S.; ISOTANI, S.M. Teste de triagem do desenvolvimento Denver II: adaptação transcultural para a criança brasileira. Com autorização do autor Frankenburg W.K. São Paulo, 2013.

SOUSA, I.C., PINHEIRO, F.B.A., MACHADO, E.T.M. A relevância da Psicoeducação familiar e o papel da família na reabilitação neuropsicológica do TEA. Braz. J. of Develop. V.7, n.3 p.22558-22570, $\operatorname{mar} 2021$.

TOMASELLO, M. Origens culturais da aquisição do conhecimento humano. São Paulo: Martins Fontes, 2003.

VIGOTSKI, L. S. A formação social da mente. São Paulo: Martins Fontes, 1998. 